\title{
A systematic review of the economic costs of gastrointestinal consequences of treatment for cancer patients and healthcare providers
}

Longo M. ${ }^{1}$, Weightman A. ${ }^{2}$, Muls A. ${ }^{3}$, Nelson A. ${ }^{1}$, Andreyev J. ${ }^{3}$, Staffurth J. ${ }^{4}$, Smith L. ${ }^{5}$, Mann M. ${ }^{1,2}$

1. Marie Curie Palliative Care Research Centre, Cardiff University, Cardiff, UK; 2. Specialist Unit for Review Evidence, Cardiff University, Cardiff, UK; 3. The Royal Marsden NHS Foundation Trust, London, UK, 4. School of Medicine, Cardiff University, Cardiff, UK, 5. Macmillan Cancer Support, London, UK

\section{Background}

Over $50 \%$ of cancer patients are expected to survive 10 years after diagnosis. Acute and chronic gastrointestinal (GI) toxicity can seriously impact patients' ability to live a normal life:

- 1 in 3 patients suffer from faecal incontinence and toilet dependency after GI surgery for a rectal cancer.

Nearly 1 in 2 patients have chronic side effects (eg diarrhoea, faecal incontinence, abdominal pain) after pelvic radiotherapy.

These toxicities reflect an economic burden to patients, healthcare providers and society as a whole.

\section{Study aim}

A systematic review of the economic costs of gastrointestinal toxicity following cancer treatment (CRD42017067800)

\section{Methods}

Grey and electronic study sources were searched. Quality assessment was carried out using an adapted CHEERS checklist.

\section{Results}

PRISMA

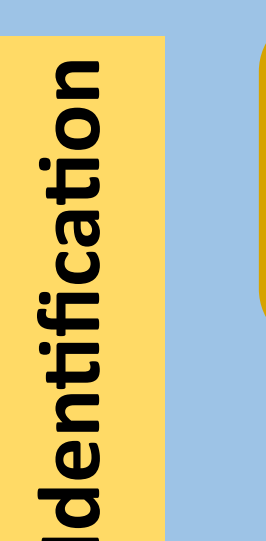

Record identified
through database
searching $(n=567)$

Additional records

identified through

other sources $(n=24)$

1

Records after duplicates removed $(n=623)$



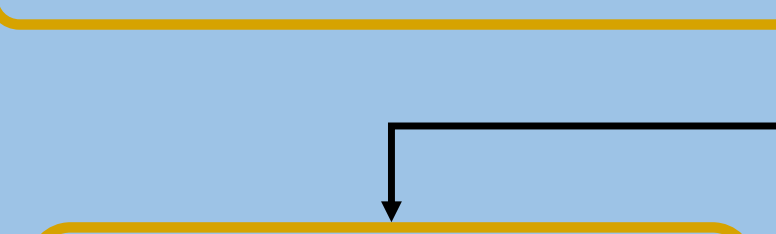

Records screened $\rightarrow$ Records excluded $(n=623)$ $(n=546)$

吾

Full-text articles assessed for eligibility $(n=77)$

Full-text articles

(ntipesin

Studies included in qualitative

health economic synthesis $(n=19$,

comprising 24 references)

$\{$ The studies were quite different.

$\{$ Large variation in sample size.

$\{$ Large variation in follow up time (from 6 weeks to 10 years).

$\uparrow$ Most studies did not differentiate between chronic and acute toxicity.

$\{$ Studies differed in methods and quality.

$\{$ Standard quality assessment checklists are difficult to apply to costs studies.

\section{Range of cost per patient ( $f$ )}

\begin{tabular}{llcc} 
Treatment & $\begin{array}{l}\text { Type of } \\
\text { cost }\end{array}$ & All Gl toxicities & $\begin{array}{r}\text { Focus on one Gl toxicity } \\
\text { (e.g. Diarrhoea) }\end{array}$ \\
Radiotherapy & Total & $£ 1,877$ to $£ 4,749$ & $f 1,758$ to $£ 1,990$ \\
\hline Chemotherapy & Monthly & $£ 192$ to $£ 380$ & $£ 16$ to $£ 902$ \\
\hline Surgery & Annual & $£ 3,864$ to $£ 3,942$ & No data \\
\hline Combi/Any & Total & $£ 1,606$ & No data
\end{tabular}

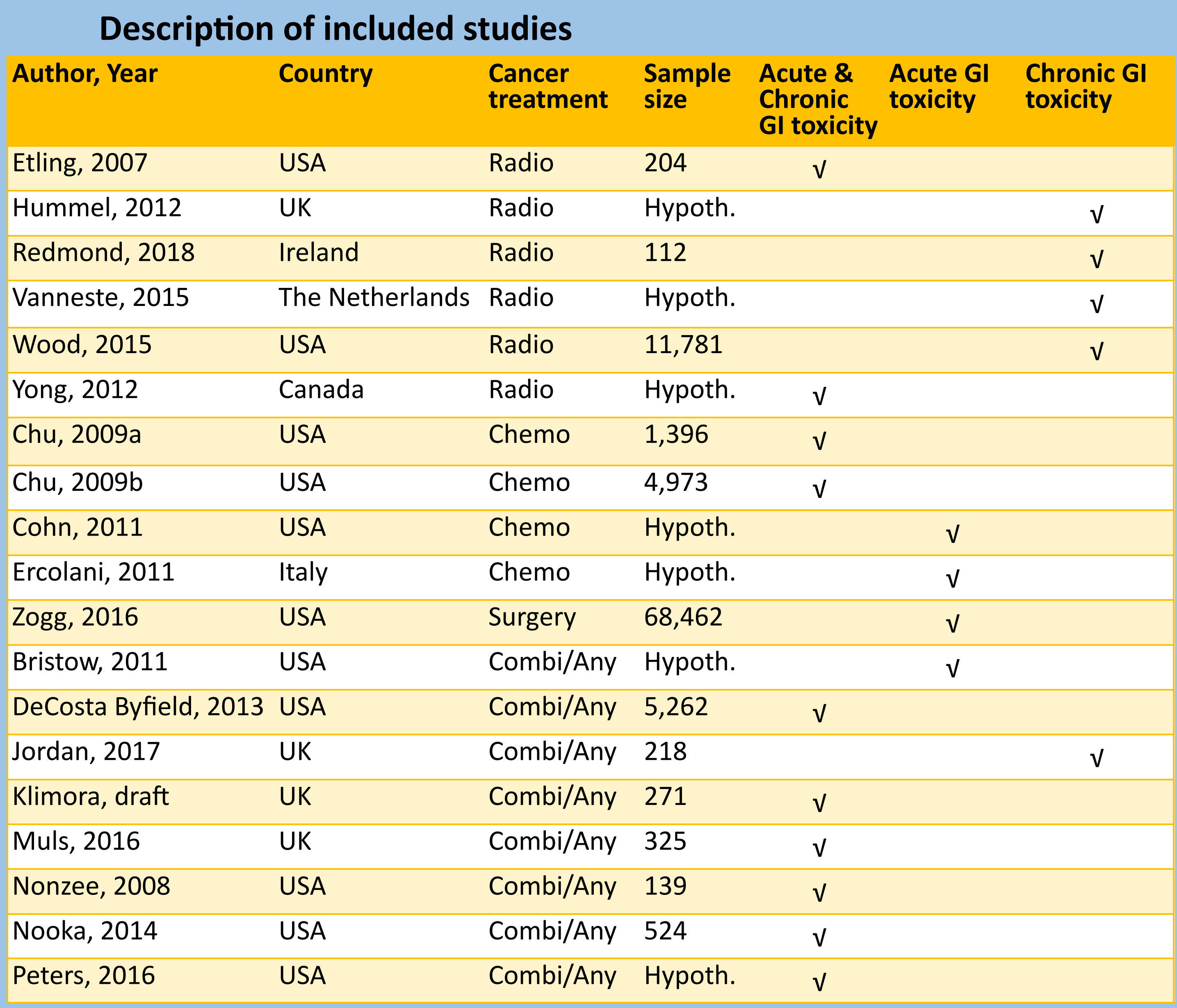

$\{$ Hospitalisation is a key cost driver.

$\$$ When available, standard deviation and range suggest a very $\checkmark$ wide distribution of costs.

$\downarrow$ There is a focus on secondary care data.

$\{$ Primary care data is very limited.

$\&$ Data on costs borne by patients, family and society was also limited. One study calculated lost income.

\section{Recommendations}

$\{$ Explore the use of screening tools to trigger earlier referral of cancer-related toxicity.

$\hat{f}$ Increase the use of routine data to study/predict long-term

\section{Conclusions}

Evidence is scarce, nonetheless it shows the need to account for both acute and chronic toxicities when comparing the cost-effectiveness of alternative cancer treatments. 